\title{
How cores grow by pebble accretion
}

\section{Direct core growth (Corrigendum)}

\author{
M. G. Brouwers, A. Vazan, and C. W. Ormel
}

\begin{abstract}
Anton Pannekoek Institute, University of Amsterdam, Science Park 904, PO box 94249, Amsterdam, The Netherlands
\end{abstract}
A\&A, 611, A65 (2018), https://doi.org/10.1051/0004-6361/201731824

\begin{abstract}
Key words. methods: numerical - planets and satellites: composition - planets and satellites: formation - planets and satellites: physical evolution - planet-disk interactions - errata, addenda
\end{abstract}

With this corrigendum we correct the erroneous vaporization curve (previously indicated as liquidus) in Fig. 6 and further clarify the discussion regarding the state of the core under the expected pressure/temperature conditions. The phase transition points in the $\mathrm{SiO}_{2}$ quotidian equation of state (QEOS) from Vazan et al. (2013) used to construct the vaporization curve were shifted to lower $P-T$ values by inaccurate scaling of the zeroenergy and bulk density parameters in their equation of state calculation. The effects of this error on our results are limited to the graph and discussion of the core-surface conditions presented in Sect. 4.2 because we did not use this equation of state in our simulations and only used it to determine the $\mathrm{SiO}_{2}$ phase at the core surface. In Fig. 1 we show the corrected figure, which replaces Fig. 6 of the paper. Figure 2, for reference, shows the corrected and erroneous QEOS curves as well as the vapor pressure and solidus.

We chose to use the simple vapor pressure formulation from Stull (1947; Eq. (33) in the paper) in our simulations to calculate vaporization rates and absorption. This expression is valid for temperatures in the range up to $4000 \mathrm{~K}$ and is therefore applicable to most of the envelope. On the other hand, the more extensive QEOS remains valid when $P$ and $T$ become large; these conditions are achieved at the core/envelope interface toward the end of our simulations. Therefore, we use the QEOS vaporization curve in Fig. 1 to assess the state of the core.

The core surface conditions are such that $\mathrm{SiO}_{2}$ does not, as we stated in the paper, reside in the vapor phase during the entire simulated period, but instead passes through different phases. We provide a brief discussion of the pebble case. In the first phase $\left(M_{\text {core }} \lesssim 0.35 M_{\oplus}\right)$, pebbles reach the core unimpeded and the core remains completely solid. Soon thereafter, the vapor pressure becomes non-negligible at the core and a fraction of the accreting $\mathrm{SiO}_{2}$ is absorbed as vapor. This is initially a small fraction, as the partial pressure of $\mathrm{SiO}_{2}$ is limited to the saturation curve set by the vapor pressure. After the core mass has grown beyond $\gtrsim 0.45 M_{\oplus}$, the pressure at the core exceeds the vapor pressure (and the QEOS curve, as they are consistent in this regime), such that $\mathrm{SiO}_{2}$ becomes gaseous. In principle, the outer core begins to evaporate partially under these $P-T$ conditions. However, our model assumes that the envelope is in quasi-hydrostatic equilibrium and that the partial pressure of $\mathrm{SiO}_{2}$ is limited to the total pressure in the

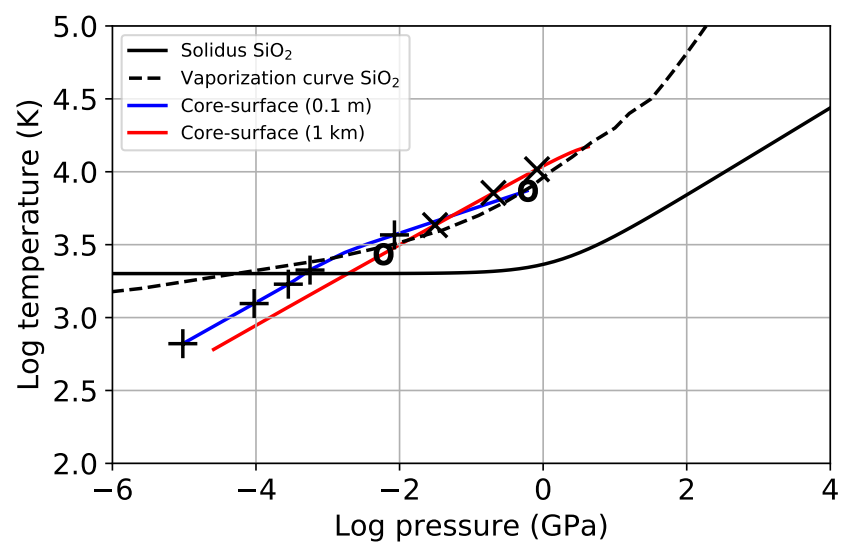

Fig. 1. Core-surface conditions during core growth by pebble and (indestructible) planetesimal accretion. The core masses in the pebble (blue line) and planetesimal (red line) cases range from $0.1 M_{\oplus}$ to $0.59 M_{\oplus}$ and 3.8 $M_{\oplus}$, respectively. The pluses on the pebble line indicate $0.1 M_{\oplus}$ core-mass intervals; the crosses on the planetesimal line indicate $1 M_{\oplus}$ intervals. The location of the $0.59 M_{\oplus}$ point is indicated by the circles. The $\mathrm{SiO}_{2}$ solidus and QEOS vaporization curve are indicated by the solid and dashed lines, respectively.

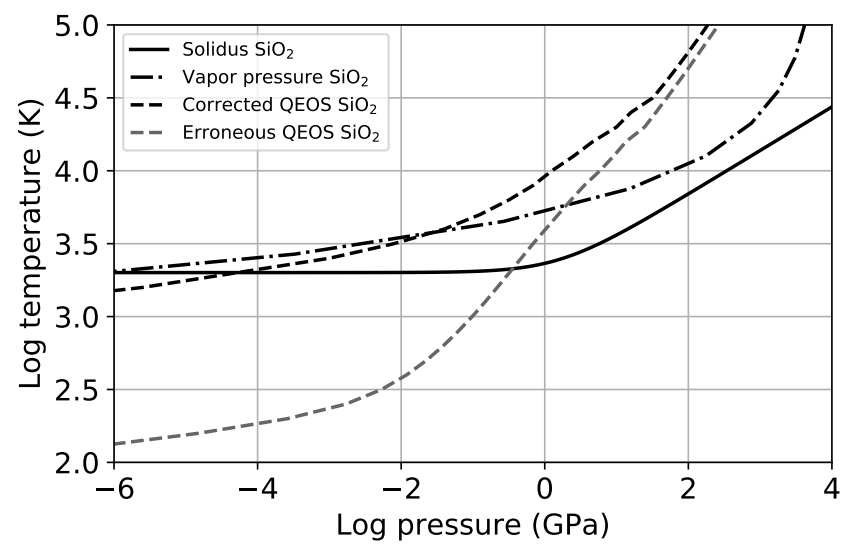

Fig. 2. $\mathrm{SiO}_{2}$ phase curve comparison. The $\mathrm{SiO}_{2}$ solidus (Poirier 2000), vapor pressure (Stull 1947), corrected QEOS, and erroneous QEOS (Vazan et al. 2013) vaporization curves are indicated by the solid, dash-dotted, and dashed lines, respectively. 
envelope, such that the fraction of $\mathrm{SiO}_{2}$ vapor reaches unity. We also assume that the enrichment is continually supplied by the pebble flux. Therefore, in our model the $\mathrm{SiO}_{2}$ at the core only evaporates when the pebble flux is insufficient to fully saturate the envelope. In the simulated period, the envelope remains fully saturated by the pebble flux, meaning that the outer core cannot evaporate and continues to grow through high- $\mathrm{Z}$ rainout.

Our simulations end when all the accreting $\mathrm{SiO}_{2}$ pebble mass can be absorbed by the envelope and therefore direct core growth is halted. We find from the QEOS that the core-surface conditions at this point are on the edge between gaseous and liquid, such that the state of the core surface is uncertain. It is possible that the outer layer of the core liquifies or evaporates during the subsequent evolution of the planet similar to the scenario described by Chambers (2017) for water planets.

\section{References}

Chambers, J. 2017, ApJ, 849, 30

Poirier, J.-P. 2000, Introduction to the Physics of the Earth's Interior (Cambridge University Press), 120

Stull, D. R. 1947, Ind. \& Eng. Chem., 39, 517

Vazan, A., Kovetz, A., Podolak, M., \& Helled, R. 2013, MNRAS, 434, 3283 